\title{
Layered 3D Printing by Tethered Pyro-Electrospinning
}

\author{
Sara Coppola $\mathbb{1}$, Giuseppe Nasti $\mathbb{1}$, Veronica Vespini $\mathbb{1}$, and Pietro Ferraro
}

Institute of Applied Sciences and Intelligent Systems ISASI CNR, Pozzuoli 80078, Italy

Correspondence should be addressed to Sara Coppola; s.coppola@isasi.cnr.it

Received 26 July 2019; Accepted 10 September 2019; Published 10 January 2020

Guest Editor: Francesco Lopresti

Copyright ( 2020 Sara Coppola et al. This is an open access article distributed under the Creative Commons Attribution License, which permits unrestricted use, distribution, and reproduction in any medium, provided the original work is properly cited.

\begin{abstract}
Nowadays it is easy to imagine that the exploitation of different additive manufacturing approaches could find use in regenerative medicine and frontiers nanotechnology with a strong interest in the development of in vivo bio-incubators that better replicate the tissue environment. Various electrospinning technologies have been exploited for the fabrication of composite polymeric architectures, where fibers have been used for the construction layer by layer of micro-architectures. Unfortunately, in case of processing biomaterials, the intrinsic factors of the materials could become obstacles when considering such advanced engineering methods. Here, for the first time, we use the pyro-EHD process for the fabrication of layered three-dimensional architectures made using a biodegradable and biocompatible polymer. The proposed approach for layered 3D printing works at mild temperature allowing deposition at high resolution and great flexibility in manufacturing, avoiding high voltage generators, and nozzles. The layered 3D printing, activated by the pyro-electric effect, is discussed and characterized in terms of geometrical features and processing parameters. Different geometries and micro-architecture (wall, square, triangle, and hybrid structures) have been demonstrated and over printing of composite polymer, obtained by mixing multiwall carbon nanotubes and fluorochrome, has been discussed, focusing on the use of a biodegradable and biocompatible polymer.
\end{abstract}

\section{Introduction}

Micro- and nanostructured fibers have found promising applications in a number of emerging fields of technology: wearable electronics, fabrication of custom-made biomedical indwelling devices, drug delivery elements, and tissue engineering scaffolds are few and very simple examples of final devices. Fibers represent the elementary component for additive manufacturing where the final object of interest is constructed through layer-by-layer structuring of the material from the bottom up $[1,2]$. The fabrication of three-dimensional (3D) structures via direct printing of fibers has been tested for a broad palette of materials (biomaterials, polymers, wax, metals, and ceramics), even if it is in case of polymeric material processing that it opens up the way to powerful applications.

Among the methods for fiber drawing, electrospinning is a well-established and simple apparatus for the production of nano and microscale fibers. Applying high voltage to a polymer solution, or a melt dispensed through a nozzle, mats of randomly positioned mesofibers are typically collected on the top of a substrate [3,4]. Starting from this concept advances in precision electrospinning methodologies, such as near-field electrospinning, electrohydrodynamic writing, and direct writing electrospinning $[5,11]$ have led to a good control for fiber placement. The properties of the architectures produced by fiber's stacking depend critically on the characteristics of the polymeric ink. The introduction of hybrid polymers, consisting in a polymeric mixture with graphene oxide $(\mathrm{GO})$ and graphene nanoplatelets (GNP) could led to create structures that can be tailored to meet specific requirements such as electrical conductivity or strength by choosing the appropriate GNP/GO ratio in case of flexible patches [12]. In conjunction with shape memory polymers (SMPs) 3D printing could be used to produce automatically deformed structures such as flexible gripper [13]. Fiber printing has been used to create tunable bistable components using SMPs consisting in special joints for twisting and rotational structures and avoiding complex and tedious post-assembly procedures [14]. In case of biomaterials, the intrinsic factors of the materials (biostability, biocompatibility, and rheology) could become obstacles when considering such advanced engineering methods for polymer processing and high temperature employed. Electrohydrodynamic (EHD) printing become a viable high resolution printing approach, in which the ink is subjected to electrostatic field to form a Taylorcone and produce a fine jet or fiber from it [15-17]. EHD printing provides greater control on fiber formation (in situ) and 
deposition by shortening the working distance and optimizing high voltage application. Both of these factors, in tandem permit precision patterning, control on fiber morphology, and the potential to build 3D structures (through over-printing). Despite all the advantages related to EHD printing, several strategies have sought to reduce the high electric field, which is characteristic of electrospinning [18-20] and the use of nozzles, introducing more delicate conditions for processing biomaterials with or without functional fillers. In fact, the EHD printing technologies are limited in their achievable structural resolution that is mainly controlled by the nozzle size and scaling down the nozzle size for better resolution will make the required filling pressure unpractical high for high viscous biopolymers [21-23].

Among these, the pyro-electrohydrodynamic (pyro-EHD) printing, where a pyroelectric material subjected to a temperature gradient is employed for drawing polymer fiber, combines mild temperature of operation with high resolution deposition, and great flexibility in manufacturing. The pyroEHD tethered electrospinning (TPES) uses a very simple set-up avoiding high voltage generators and has been already successfully tested for the fabrication of planar scaffold for tissue engineering and microfluidic devices [24, 25]. The dimension of the printed fibers overcomes the limit of conventional EHD where the achievable structural resolution is mainly controlled by the nozzle size, drawing fibers directly from the elongation of the Taylor cone. Here, for the first time, we use the pyro-EHD process for the fabrication of layered three-dimensional architectures made using a biodegradable and biocompatible polymer. The $3 \mathrm{D}$ pyro-printing is discussed and characterized in terms of characteristics and processing parameters. Different geometries and micro-architecture (wall, square, triangle, and hybrid structures) have been demonstrated and over printing of composite polymer, obtained by mixing multiwall carbon nanotubes and fluorochrome, has been discussed.

\section{Materials and Methods}

2.1. Material. Poly(lactic-co-glycolic acid) PLGA (38,000 to 54,000 Da; PLGA Resomer RG 504 H, Boehringer Ingelheim) and dimethyl carbonate (DMC 20-30w/v, DMC 99\%) were purchased from Sigma-Aldrich and used without further purification. Multiwalled carbon nanotubes (MWCNT, $d=110-170 \mathrm{~nm}, \quad$ length $=5-9 \mu \mathrm{m}, \quad$ purity $>90 \%)$ were purchased from Sigma-Aldrich and used without further purification. In some experiments a fluorocrome (Nile red, $\mathrm{C}_{20} \mathrm{H}_{18} \mathrm{~N}_{2 \mathrm{O} 2}$, technical grade, Sigma-Aldrich, and $2 \mu \mathrm{g}$ for milligram of PLGA) was added to the polymeric solution and used as model drug.

2.2. Solution Preparation. The polymeric solutions consisting in PLGA dissolved in DMC were prepared using a fixed concentration of $25 \mathrm{wt} \%$ mixing a proper amount of the polymer and solvent at $70^{\circ} \mathrm{C}$ under magnetic stirring for 3 hours. In case of MWCNTs in PLGA solution, the filler was added before stirring for 3 hours at concentration of $20 \mathrm{wt} \%$ and then the solution was properly mixed and sonicated before use for $4 \mathrm{~h}$ using a low-mid intensity amplitude and a duty cycle of $10 \%$ (ON time per cycle) in order to break nanoparticles' aggregates. The fluorocrome (Nile Red) was added to the PLGA solution during the preparation of the solution and mixed under magnetic stirring for 3 hours at $70^{\circ} \mathrm{C}$. All the solutions for fiber stacking were tested in air at ambient temperature.

The TPES was activated by the thermal stimulation of a lithium niobate crystal (LN, $c$-cut, both sides polished, $500 \mu \mathrm{m}$ thick, and Crystal Technology Inc.). A commercial glass microslide is used as receiving target (thickness $100 \mu \mathrm{m}$ ) after washing and cleaning through Ethanol (from Sigma-Aldrich).

2.3. Fiber Drawing. The tethered pyro electrospinning is employed for the fabrication of polymeric fibers. A small drop of polymeric solution is used as reservoir and deposited under the LN crystal plate used for the activation of the TPES process, as described in detail in the "Tethered pyro-electrospinning of 3D microstructres" paragraph. The reservoir drop was directly pipetted over a hydrophobic micropillar made in Polydimethylsiloxane (PDMS) and placed over a commercial microscope glass slide. Under the activation of the TPES process, the droplet started to deform, creating an elongated tip from which fibers were drawn. The target used for the collection was placed in front of the drop and removed at the end of the over-printing procedure. The microstructures were analysed and characterized using a microscope Zeiss AXIO Zoom.V16.

2.4. Morphological Analysis. Scanning electron microscopy (SEM) analysis was performed by means of a FEI Quanta 200 FEG (FEI, Eindhoven, The Netherlands) microscope using an acceleration voltage of $10-20 \mathrm{kV}$. Images were collected with a secondary electron detector. 3D micro-samples were sputtercoated with a 5-10 nm thick Au-Pd layer.

\section{Results and Discussion}

3.1. Tethered Pyro-Electrospinning of 3D Microstructres. The over printing obtained through TPES is based on the exploitation of the intense pyroelectric field emerging from a LN crystal when subjected to a temperature gradient. The equipment used for the pyro 3D-printing (Figure 1(a)) consists of a reservoir solution drop, a pyroelectric crystal, a thermal control system, a programmable $X-Y$ stage motion, glass target holder and various manual micro-stages with $X-Y-Z$ axis control. Once the crystal is heated by contact using a conventional soldering iron tip, the pyroelectric material, subjected to a temperature gradient of about $80^{\circ}$, creates an high electric field. The intensity of the pyro-electric field is primarily function of the pyro-electric coefficient. In fact, when the thermal control system locally heats the crystal, a sudden surface density charge $\sigma$ immediately appears given by $\Delta \sigma=p \Delta T$ neglecting losses, where $p$ is the material-specific pyroelectric coefficient $\left(P c=-8.3 \times 10^{-5} \mathrm{C} /{ }^{\circ} \mathrm{C} / \mathrm{m}^{2}\right.$ for $\mathrm{LN} @$ $\left.25^{\circ} \mathrm{C}\right)$.

Once the pyro-electric field is on, a large voltage is applied between the LN crystal and the plate supporting the drop, which would gather mobile ions in the printing solution near 


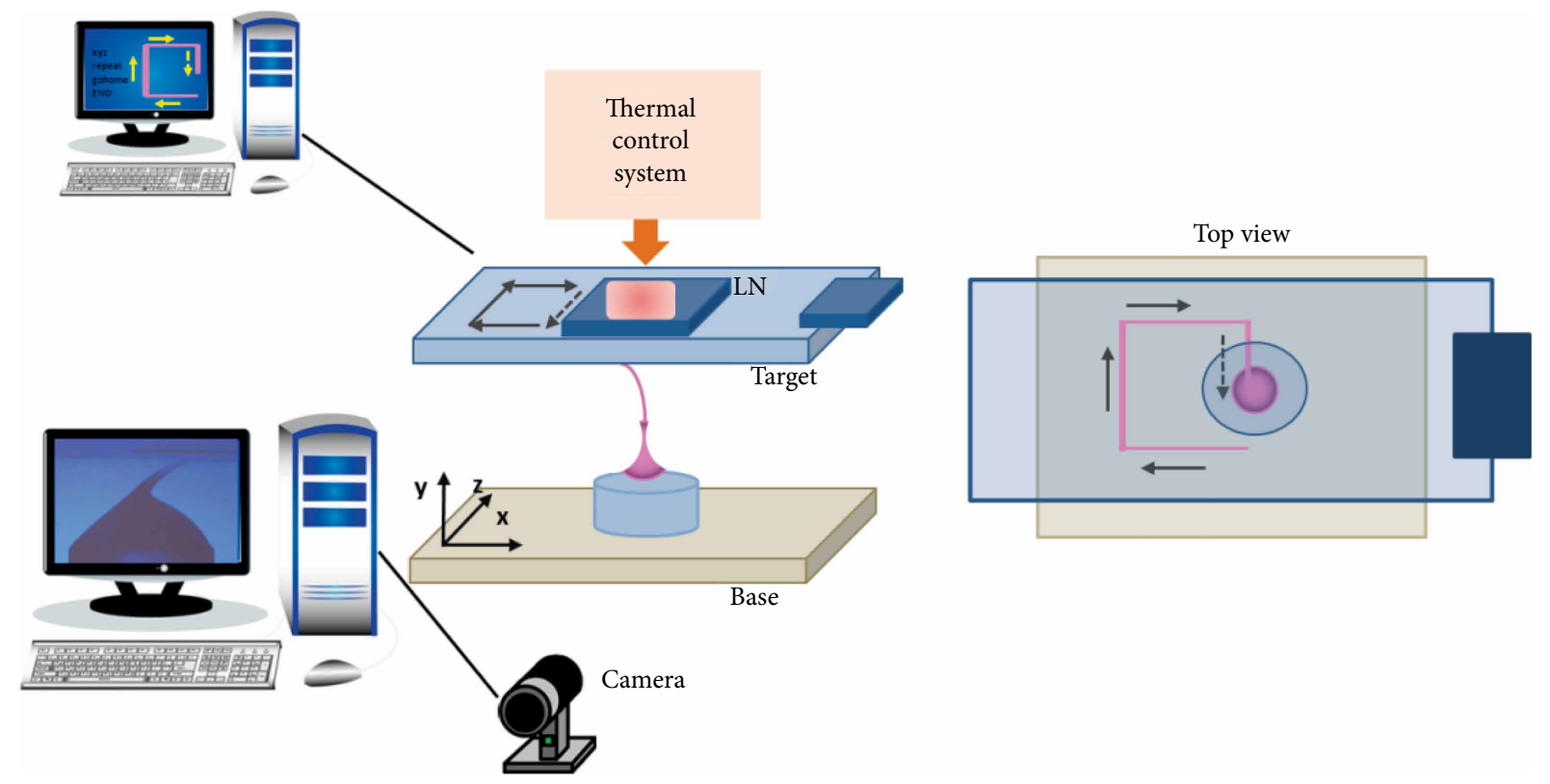

FIGURE 1: Outline of the set-up for layered 3D printing: the reservoir drop is sited under the LN crystal heated by a thermal control system. During the experiment real time visualization was ensured in order to control at the same time the jetting process and the target position. For the fabrication of layered architecture the target was moved along the $x, y$ direction. In the top-view the pink trails and the arrows indicate the direction of movement.

the surface of the starting drop to form chargers. The charges would bring an electrostatic force to deform the drop's meniscus to form a conical shape (Taylor cone). When the electrostatic force will overcome the surface tension at the Taylor cone, a jet would be injected from the meniscus onto the receiving substrate. The reservoir droplet is sited in front of the active crystal at a distance $\mathrm{h}<1 \mathrm{~mm}$. The selected distance $h$ allows preventing the jetting from buckling as it hits the target. In this way, the bending instability and splitting of the charged jet are overcome. The movement of substrate mainly reduces the accumulated charge to improve the stability of pyro-electrospinning at low speed, and primarily draws the fibers when the speed reaches the critical value of jetting. The laws that rule the conventional electrospinning process are still effective in case of the TPES, while the main difference lies in the exploitation of pyro-electricity to activate the jetting. The jet diameter during electrospinning is also dependent on surface tension $\gamma$, flow rate $Q$, dielectric constant $\varepsilon$, and electric current $I$ in the jet. Fridrikh presented a simple analytical model to calculate the diameter of the fibers produced by electrospinning [26].

$$
R=\left(\gamma \varepsilon \frac{Q^{2}}{I^{2}} \frac{2}{\pi(2 \ln \Psi-3)}\right)^{1 / 3} .
$$

The parameter $\Psi \sim R / h$ is the dimensionless wavelength of the instability responsible for the normal displacements. The distance $h$ is able to affect the solidification degree of the fiber at the time of reaching the substrate. Mostly, the plotting results will be affected by the fluid properties of the ink, printing process conditions (i.e., ink temperature, standoff distance), and applied printing temperature. In our experiments the PLGA/DMC solution at a concentration of $25 \mathrm{wt} \%$ was chosen because of the good printing properties for the fabrication of scaffold for tissue engineering. Ambient parameters, such as the humidity and temperature of the surroundings, play a significant role in discharge, solidification, evaporation rate, and viscosity of the printed fibers, so a microenvironment should be integrated to improve the repeatability.

A small sessile drop of the polymer solution is casted on micro pillar (diameter of around $500 \mu \mathrm{m}$ ), lying in front of the pyroelectric crystal and fixed to moving translator in order to control the vertical position. Above the sessile drop, a thin microscope glass slide ( $\sim 150 \mu \mathrm{m}$ thickness) is fixed to a twoaxis micro-translators (PI, Mercury C-863 servo controller) controlled by the computer software. By controlling the movement of the translators is possible to define the trail of different geometries, the 3D micro-structures would be fabricated by direct overlay of the emitted fiber without jetting interruption. In order to follow the evolution of direct writing in real time, an USB camera is focused on the side view of the region of interest, including the target substrate and the reservoir drop. During the printing process, the starting drop was fixed, while the target was moved in plane along the direction required for the 3D fabrication. The top view of Figure 1 shows the movement in plane along the $x$ and $y$ directions, the pink lines represent the printed track and the arrows indicate the movement directions. A high speed camera is sometimes required to monitor the dynamic process of feature formation, deposition, and stacking, which would generate new insights into directing the optimization of process parameters as well as deeply understand the pyro-electrohydrodynamic process.

3.2. Process Parameters. During the printing process by TPES, fibres were laid down on the glass substrate and the microstructures were obtained by superimposition. Fibre 
characteristics such as fibre diameter and its uniformity were adjusted by varying several controllable process parameters. In this study, three parameters were considered to have major effects on fibre diameter as well as morphology, fixing the temperature of operation for the activation of the pyroelectric effect at about $110^{\circ} \mathrm{C}$, the volume of the reservoir drop, the velocity of the moving stages, and the working distance between the base supporting the reservoir drop and the receiving substrate. The resulting printed fibers had a diameter $(d)$ ranging between $10 \mu \mathrm{m}<d<30 \mu \mathrm{m}$. Controlling these parameters could be possible to produce fiber of about $1 \mu \mathrm{m}$ diameter or even less, as demonstrated in case of TPES.

\subsection{Design of 3D Micro-Structures. Pre-designed pattern} models were created controlling the motion directions of the stages so that the fibers could stack on top of each other creating a multilayer structure. A high resolution moving stage is required to regulate the arrangements of the printed features and consequently to determine the patterns. In Figure 2 we reported a schematic overview of the different microstructures that were realized using the proposed approach. We started from the fabrication of a straight wall (Figure 2(a)), the elongated fibers were printed one over the other by superimposition, the blue arrows indicated the direction of moving on the plane $(x, y)$ while the fibers were stacked along the $z$ direction. In the supplementary movie \# 1 is reported the overprinting procedure in the simpler case for creating a wall. Then we proceeded to the design of a square where only one side was superimposed by direct printing. In Figure 2(b), the lines in grey were printed just one time while the dotted one in orange was realized in a second step by superimposition, creating an higher side. We tested the properties of 3D printing in case of a square geometry created side by side. Starting from the construction of the first side, obtained by the overlay of 5 fibers, we moved to the adjacent side realizing it with the same number of layers and we replicated it for the remaining sides. In the layout of Figure 2(c), the red arrow suggests the side that was constructed by superimposition, one side after the other. The dotted orange lines represent the designed lines that are going to be constructed in a clockwise direction. We demonstrated the fabrication of square or triangle geometry also by the overlay of the complete track (Figures 2(d) and $2(\mathrm{e})$ ). We printed the outline of interest (square or triangle) and then rewrote the same profile more times, one layer over the other up to the final height of the microstructures.

3.4. Fabrication of 3D Micro-Structures. For the experimental part of our work we used the PLGA/DMC solution doped with a fluorochrome. The PLGA could be dissolved in a range of common organic solvents, DMC was chosen as solvent because it easily dissolves PLGA and evaporates rapidly, matching operational time needed for droplet deformation, and fiber elongation. Moreover, DMC is biodegradable and less toxic than other conventional organic solvents and it has been widely used for biomedical applications (scaffold, nano/ microsphere, drug delivery systems). The biodegradation rate of the PLGA could be also tailored because it is function of the molar ratio of the lactic and glycolic acids in the polymer chain, by controlling these parameters it would be possible to manage and adopt it for different case uses. The solution of PLGA in DMC would be transparent so that for improving the visualization in real time of the jet elongation and its deposition we added a fluorocrome emitting in the visible range. The printed fibers appears pink when analysed thorough the optical microscope. Moreover, the addiction of a filler, could be considered as a starting test related to the use of active components that could be added to the solution and delivered with an appropriate rate, in case of drug delivery routines. It is important to point out that its addiction to the solution did not affect the jet deformation under the action of the pyro-electric effect and the printing properties. For the microstructures' fabrication we started form the construction of a single wall where the printed fiber was conceived as a building block. Similar to the conventional additive manufacturing approaches, the final object is constructed by adding layer-upon-layer of polymeric material, in our case the polymeric fiber ejected without interruption. Even if the working distance was defined in a proper working range, in order to avoid the bending and buckling instabilities of the ejecting fiber, the overlay was simple in case of a single wall while become more critical for complex structures. Figure 3(a) shows a stereomicroscope image of a multi-layered wall consisting in about 10 layers with good superimposition and homogenous stacking, no spaces or defects are visible. In order to better observe the stacking of the polymeric layer, a SEM characterization was included consisting in the top view of the printed wall with two different angles of observations, Figures 3(b) and 3(c). In both cases the defects appeared minimal and the fibers were stacked with high precision one over the other with no evident slight errors.

Following the demonstration of the simple printed wall we went through the fabrication of more complex geometries. In particular, we focused on the fabrication of a polymeric grid were only one of the horizontal line crossing two vertical ones was over layered. The procedure for the fabrication was outlined in Figure 2(b), the vertical fibers were directly printed over the glass slide, then the sample was rotated of $90^{\circ}$ in order to print the intercepting lines, one of these consisted in a single layer while the second one was overprinted 4 times. In Figure 4 the outline of the fabrication is reported with the corresponding stereomicroscope and SEM images. The resulted square had the front side higher than the remaining perimeter. The horizontal fibers of Figure 4(c) were thinner than the vertical ones and they were partially suspended at the intersection of the square, as clearly visible in the SEM image.

As described in the scheme of Figure 2(c), the next microstructure that was fabricated consisted in a square with all the side made by the same number of layer. Each side was made of 5 layers and it was completed before moving to the adjacent one. The fabrication procedure presented a clear mismatch in correspondence of the angles between two adjacent sides, probably this defect was due to the fast change in velocity of the moving translator between perpendicular direction that was not followed at the same rate by the polymer jet deformation. This mismatch is also accentuated because the PLGA/ DMC solution was integrated with MWCNTs improving the stability of the jet as demonstrated in a previous work but, 
(a) Single wall srtanding alon over the substrate

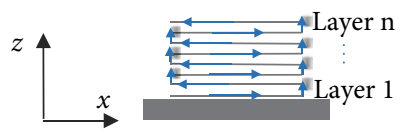

(b) Single wall crossing 2 parallel fibers
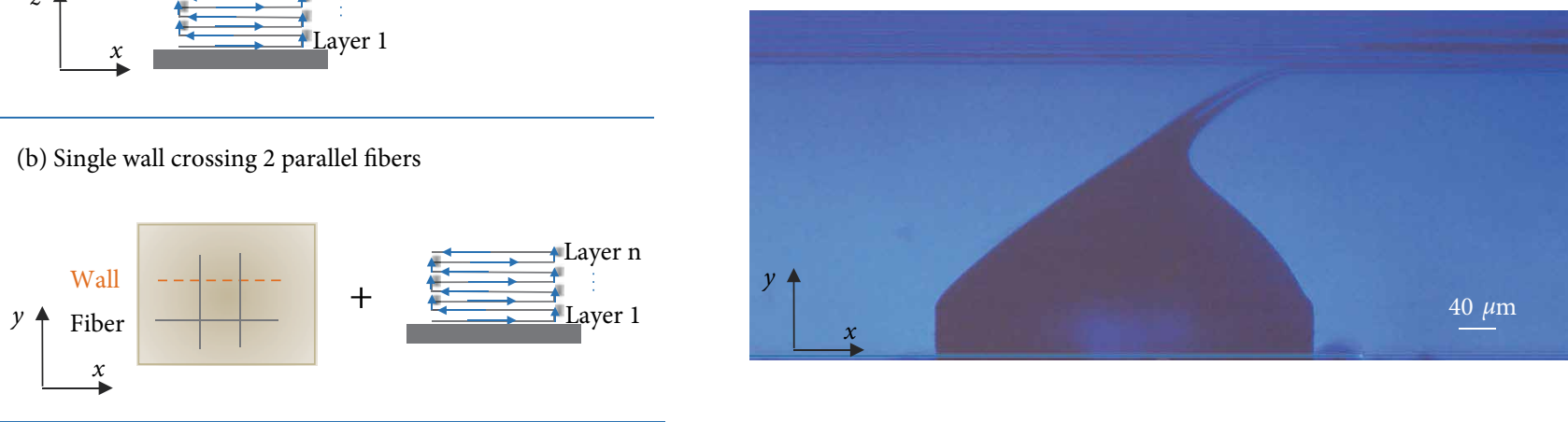

(c) Cubic architecture fabricated side by side

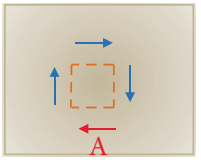

Side A

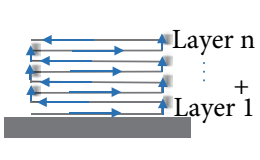

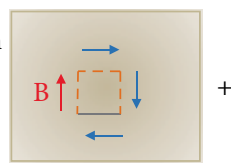

Side B

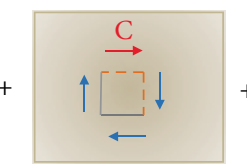

Side C

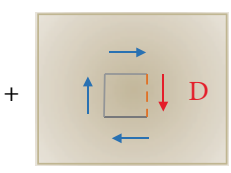

Side D

(d) Cubic architecture fabricated overwriting the square profile 5 times

(e) Triangular architecture fabricated overwriting the triangle profile 5 times
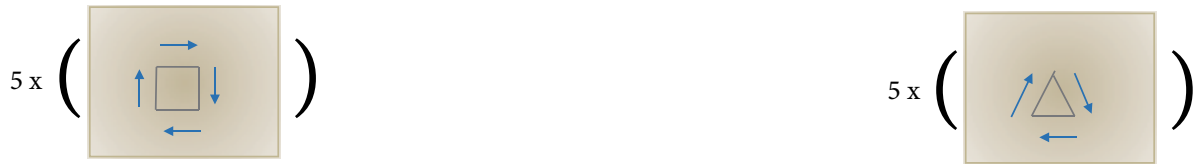

FIGURE 2: Schematic outline of three dimensional architectures: (a) a single wall free standing along the $z$ direction was constructed by overlay, the blue arrows indicate the direction of printing, (b) a single wall was constructed crossing a matrix of single fibers, creating a sort of square profile where only one side was obtained by superimposition, (c) a cubic architecture was constructed side by side, starting from the fabrication of the first wall (side $A$ ) all the adjacent sides were completed in a clockwise direction, and (d, e) cubic and triangular architecture were obtained by overlay of the complete profile, starting from the first track, the same profile was overwritten. The blue arrows indicated the direction of printing.

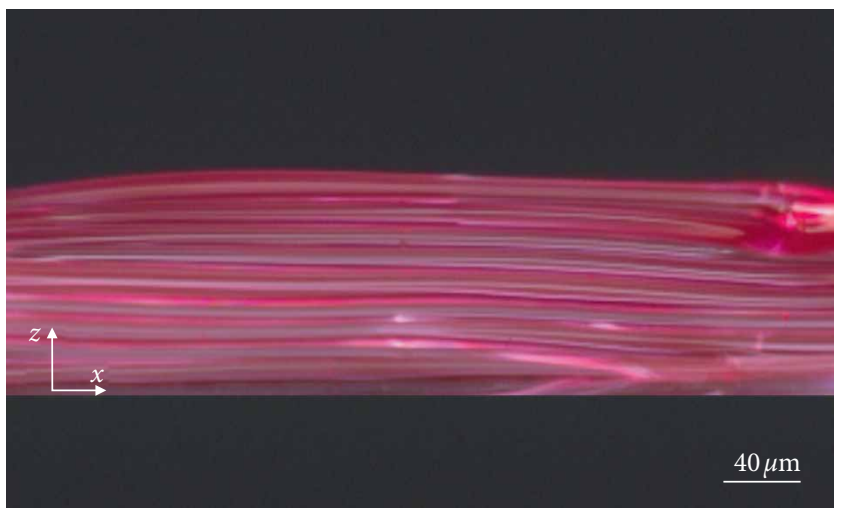

(a)

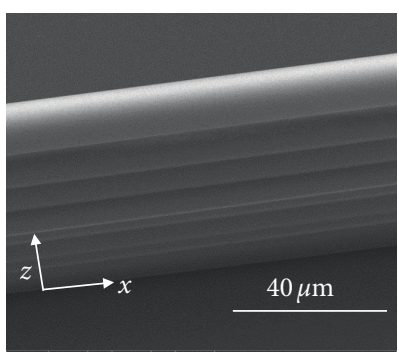

(b)

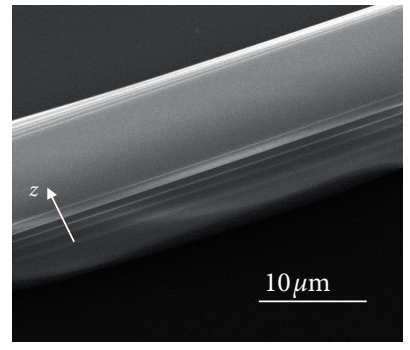

(c)

FIGURE 3: (a) Free standing single wall, (b-c) SEM image of the multi-layered wall with two different angle of observation.

reducing the ability to adapt with a fast response to instantaneous change of direction (i.e., switching from one side to the other implies a change in direction of $90^{\circ}$ ). In Figure 5 the outline of the square and two different sides are showed, out of focus is possible to distinguish the complete profile. The image is out of focus because is taken by tilting the sample observed using the stereomicroscope in order to give a three-dimensional visualization of the overprinted side. 


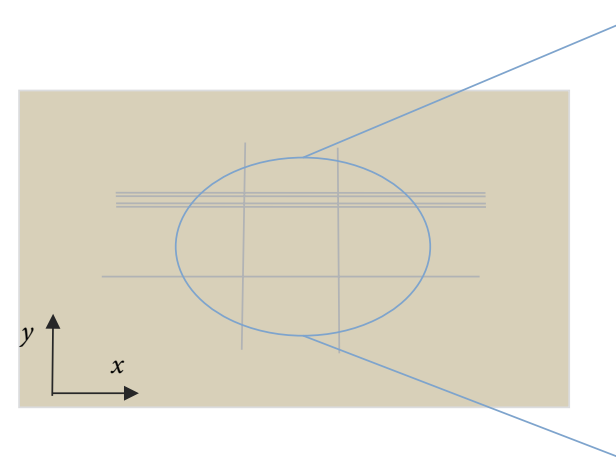

(a)

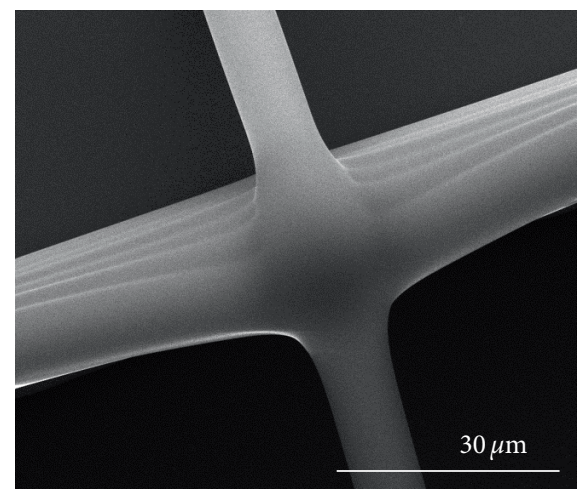

(c)

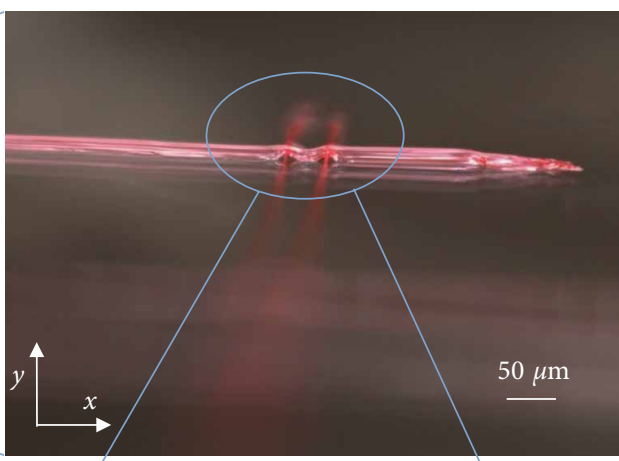

(b)

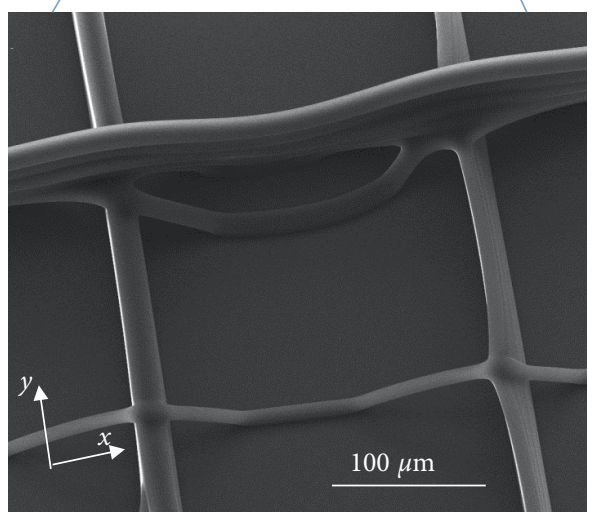

(d)

FIgURE 4: (a) Schematic outline of the experiment. (b) Streomicroscope image of a single wall crossing a matrix of single fibers at the bottom. (c) Close up of the crossing and focus on a crossing point making evident the good control in superimposition (d).

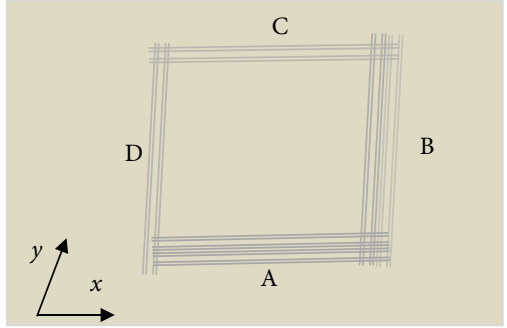

(a)

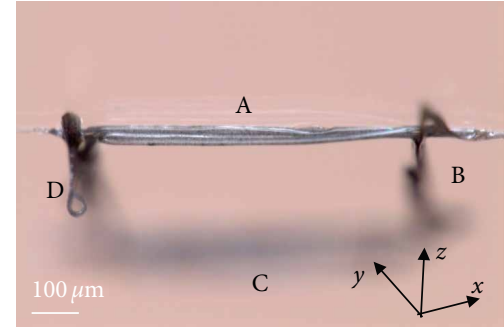

(b)

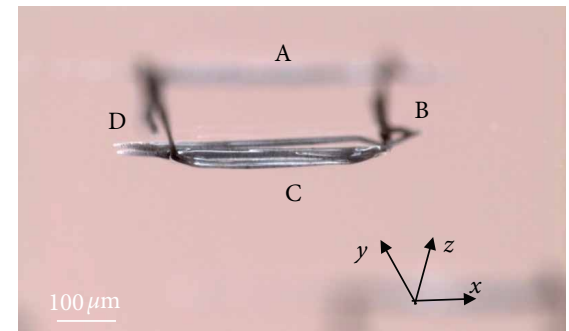

(c)

FIgURE 5: (a) Schematic outline of the experiment. (b) Stereomicroscope image of side A where is possible to reconstruct out of focus the complete profile, changing the point of view and titling the sample during the observation it is possible to analyse the opposite profile (side $\mathrm{C})$, reconstructing partially out of focus the remaining profile (c).

In order to realize a more regular square structure, following the instruction of Figure 2(d), we wrote by direct writing a cubic architecture with a square base. Differently from the construction side by side, in this experiment the complete profile was superimposed for 5 times. The resulting microstructure appears more regular, the fibers overlaid with high precision all over the sides and in particular in correspondence of the angles. In order to achieve a more evident three-dimensional image of the micro-structure, the sample was tilted at different angles under the stereomicroscope. The resulting images were acquired to collect a close-up of the intersection between two adjacent sides and the overview of a single side, the leftover profile was out of focus consequently to the tilting of the sample respect to the visualization line of the microscope (Figure 6).

Following the same procedure for the realization of a regular three-dimensional microstructures we tested a triangular geometry where the angles between the adjacent side are acute $\sim 30^{\circ}$. The construction of the architecture by superimposing the complete profile three times resulted in a good spatial resolution and vertical finishing. In Figure 7 we reported various points of view obtained by tilting the substrate during the observation. The long distance image gave the idea of the total microstructure, while the tilted images reported one side in focus with the remaining profile visible in perspective out of focus. 


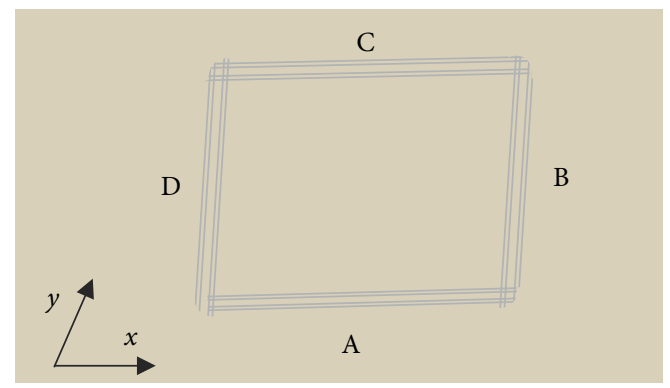

(a)

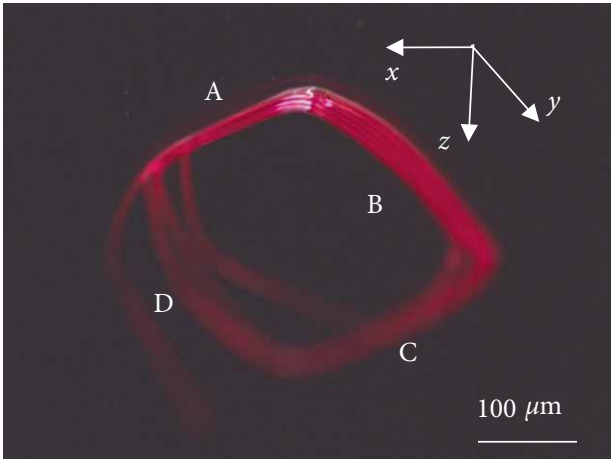

(c)

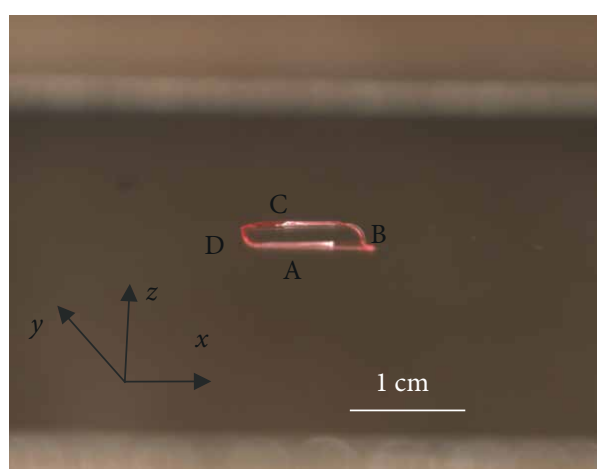

(b)

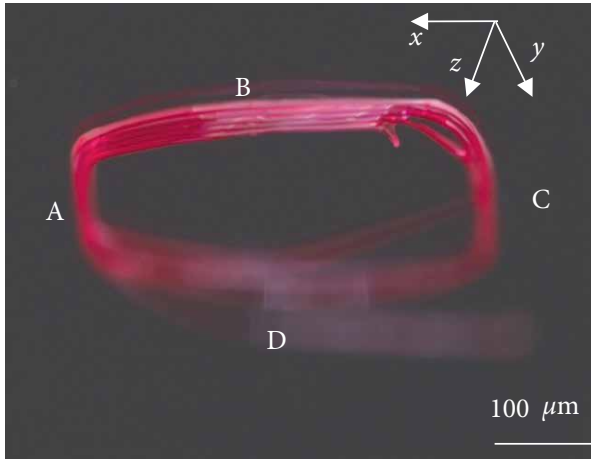

(d)

FIGURE 6: (a) Schematic outline of the cubic microstructure. (b) Stereomicroscope image of the complete architecture where every side was named $(A, B, C, D)$. (c) Changing the point of view and titling the sample during the observation it is possible to analyse the layered angle constructed between two adjacent sides. (d) One of the side of the microstructure (side B) reconstructing partially out of focus the remaining profile.

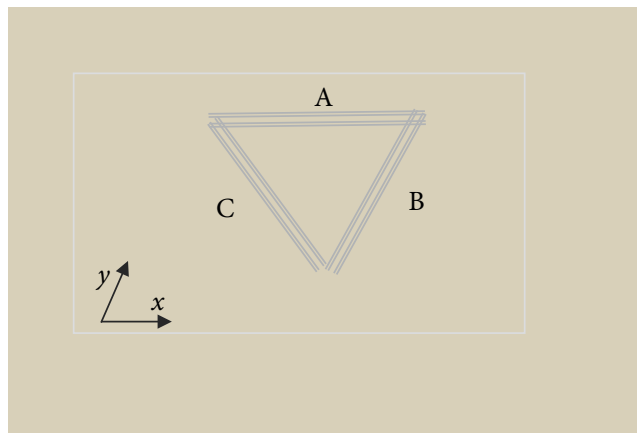

(a)

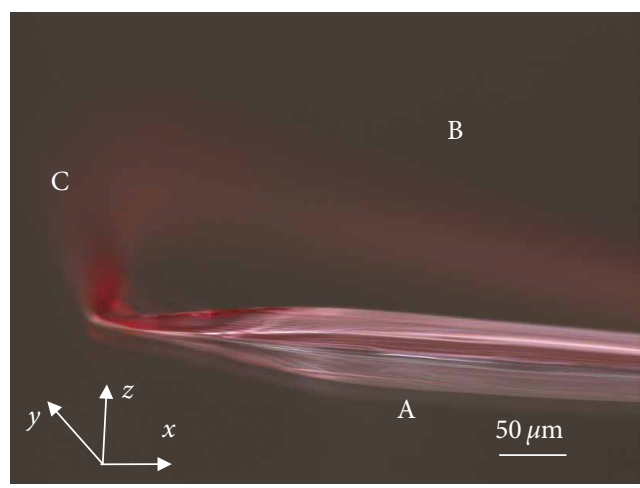

(c)

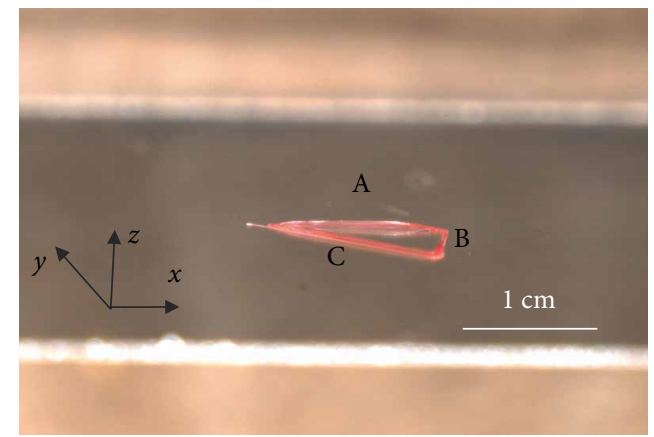

(b)

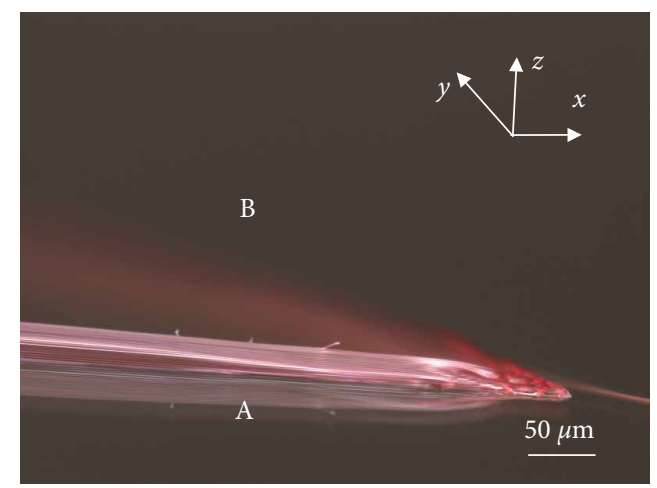

(d)

FIGURE 7: (a) Schematic outline of the triangular microstructure. (b) Stereomicroscope image of the complete architecture where every side was named (A, B, C). (c) Changing the point of view and titling the sample during the observation it is possible to analyse the multi-layered profile. (d) One of the side of the microstructure (side A) reconstructing partially out of focus the remaining profile and the other side B. 

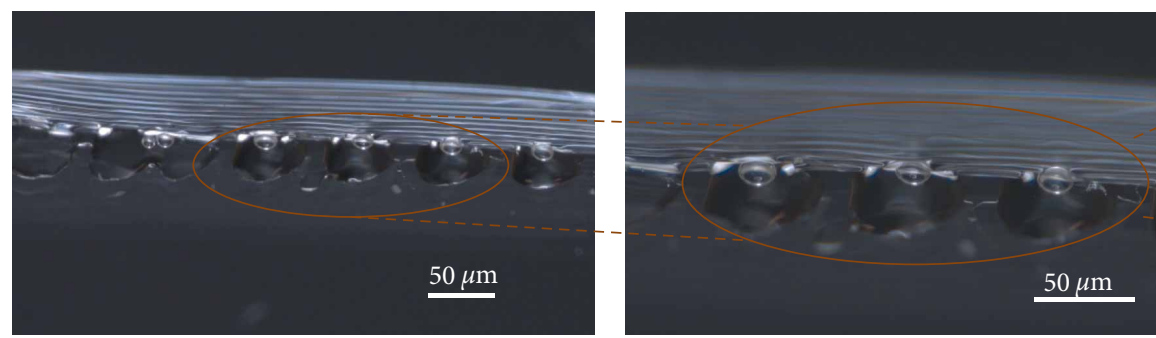

(a)

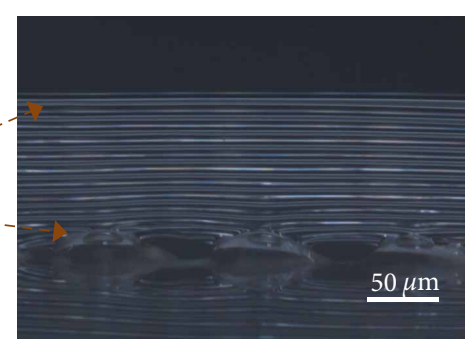

(b)

FIGURE 8: (a) Hybrid microstructure made of hemispherical drops and multi-layered fibers. The drops were used as pillars for the construction of a free standing wall. $(b, c)$ Stereomicroscope image and close up of the sustaining pillars and of the overprinted fibers.

In the last experiment reported in Figure 8 we showed how was possible to combine different modalities of TPES for the fabrication of more complex structures. We started with the direct printing of polymer drops. The substrate was not coated and the dispensed drop slightly collapsed after printing assuming a hemispherical shape. Once produced an array of separated drops we used them as pillars for the construction of a polymeric wall. We superimposed the polymeric fiber twenty times demonstrating the fabrication of a stable multilayer structure, higher than the previous example proposed. Figure 8(a) shows the combined microstructure, while Figures $8(\mathrm{~b})$ and $8(\mathrm{c})$ show the close-up of the two components, the hemispherical pillars and the multi-layered wall.

\section{Conclusions}

The system proposed based on the exploitation of the TPES and allows to create three dimensional microstructures. For the first time, fibers drawn by the action of the pyro-electric field are used as building blocks for additive manufacturing. The proposed approach for layered 3D printing works at mild temperature allowing deposition at high resolution and great flexibility in manufacturing. The pyro-EHD tethered 3D printing uses a very simple set-up avoiding high voltage generators and nozzles. The experiments reported furnish a starting point for the implementation of the proposed technique. In fact, starting from the fabrication of elementary geometry (wall, cubic, and triangular microstructures) this work represents the base for the design of more complex micro architectures. The fabrication process is described in details; the fabricated microstructures have been characterized, focusing on the use of composite material and, in particular, of biocompatible and biodegradable biomaterials. Taken into account that there is a growing demand for novel products and devices such as encapsulation systems, it is easy to imagine that the exploitation of different additive manufacturing approaches could find use in regenerative medicine with a strong interest in the development of in vivo bio-incubators that better replicate the tissue environment.

\section{Data Availability}

The data used to support the findings of this study are included within the article.

\section{Conflicts of Interest}

The authors declare that there is no conflict of interest regarding the publication of this paper.

\section{Funding}

We acknowledge financial support from the project PM3Piattaforma Modulare Multi-Missione under the National Research Program PON 2014-2020 (ARS01_01181).

\section{Supplementary Materials}

In the supplementary movie \#1 is reported the overprinting procedure in the simpler case for creating a wall. (Supplementary Materials)

\section{References}

[1] C. Wei and J. Dong, "Hybrid hierarchical fabrication of threedimensional scaffolds," Journal of Manufacturing Processes, vol. 16, pp. 257-263, 2014.

[2] Y. Han, C. Wei, and J. Dong, "Super-resolution electrohydrodynamic (EHD) 3D printing of micro-structures using phase-change inks," Manufacturing Letters, vol. 2, pp. 96-99, 2014.

[3] N. Bhardwaj and S. C. Kundu, "Electrospinning: a fascinating fiber fabrication technique," Biotechnology Advances, vol. 28, pp. 325-347, 2010.

[4] A. Greiner and J. H. Wendorff, "Electrospinning: a fascinating method for the preparation of ultrathin fibers," Angewandte Chemie International Edition, vol. 46, no. 30, pp. 5670-5703, 2007.

[5] G. Luo, K. S. Teh, Y. Liu, X. Zang, Z. Wen, and L. Lin, "Directwrite, self-aligned electrospinning on paper for controllable fabrication of three-dimensional structures," ACS Applied Materials \& Interfaces, vol. 7, no. 50, pp. 27765-27770, 2015.

[6] J. He, F. Xu, Y. Cao, Y. Liu, and D. Li, “Towards microscale electrohydrodynamic three-dimensional printing," Journal of Physics D: Applied Physics, vol. 49, no. 5, p. 055504, 2016.

[7] J. He, F. Xu, R. Dong, B. Guo, and D. Li, "Electrohydrodynamic $3 \mathrm{D}$ printing of microscale poly ( $\varepsilon$-caprolactone) scaffolds with multi-walled carbon nanotubes," Biofabrication, vol. 9, no. 1, p. $015007,2017$. 
[8] D. Sun, C. Chang, S. Li, and L. Lin, "Near-field electrospinning," Nano Letters, vol. 6, no. 4, pp. 839-842, 2006.

[9] H. Chen, A. de B. F. B. Malheiro, C. Van Blitterswijk, C. Mota, P. A. Wieringa, and L. Moroni, "Direct writing electrospinning of scaffolds with multidimensional fiber architecture for hierarchical tissue engineering," ACS Applied Materials o Interfaces, vol. 9, no. 44, pp. 38187-38200, 2017.

[10] A. Hrynevich, B. S. Elçi, J. N. Haigh et al., "Dimension-based design of melt electrowritten scaffolds," Small, vol. 14, no. 22, p. 1800232, 2018.

[11] F. M. Wunner, P. Mieszczanek, O. Bas et al., "Printomics: the high-throughput analysis of printing parameters applied to melt electrowriting," Biofabrication, vol. 11, no. 2, p. 025004, 2019.

[12] J. J. Moyano, A. Gómez-Gómez, D. Pérez-Coll, M. Belmonte, P. Miranzo, and M. Osendi, "Filament printing of graphenebased inks into self-supported 3D architectures," Carbon, vol. 151, pp. 94-102, 2019.

[13] A. Tong, J. Y. Hu, M. H. Zhang, Y. Jin, and J. K. Du, "Flexible gripper manufacturing and simulation based on 3D printing," in Symposium on Piezoelectrcity Acoustic Waves and Device Applications (SPAWDA), IEEE, Harbin, China, 2019.

[14] H. Y. Jeong, S.-C. An, I. C. Seo et al., "3D printing of twisting and rotational bistable structures with tuning elements," Scientific Reports, vol. 9, no. 1, 2019.

[15] S. Vijayavenkataraman, S. Thaharah, S. Zhang, W. F. Lu, and J. Y. H. Fuh, "Electrohydrodynamic jet 3D-printed PCL/PAA conductive scaffolds with tunable biodegradability as nerve guide conduits (NGCs) for peripheral nerve injury repair," Materials \& Design, vol. 162, pp. 171-184, 2019.

[16] H. Liu, S. Vijayavenkataraman, D. Wang, L. Jing, J. Sun, and $\mathrm{K}$. He, "Influence of electrohydrodynamic jetting parameters on the morphology of PCL scaffolds," International Journal of Bioprinting, vol. 3, no. 1, pp. 72-82, 2017.

[17] Y. Han and J. Dong, "Electrohydrodynamic printing for advanced micro/nano manufacturing: current progresses, opportunities, and challenges," Journal of Micro and NanoManufacturing, vol. 6, no. 4, 2018.

[18] S. A. Harfenist, S. D. Cambron, E. W. Nelson et al., "Direct drawing of suspended filamentary micro- and nanostructures from liquid polymers," Nano Letters, vol. 4, no. 10, pp. 19311937, 2004.

[19] X. Li, L. Wang, G. Ma et al., "Low-voltage continuous electrospinning patterning," ACS Applied Materials \& Interfaces, vol. 8, no. 47, pp. 32120-32131, 2016.

[20] Z. Li, J. Tuffin, I. Lei et al., "Solution fibre spinning technique for the fabrication of tuneable decellularised matrix-laden fibres and fibrous micromembranes," Acta Biomaterialia, vol. 78, pp. 111-122, 2018.

[21] Y. A. Huang, N. Bu, Y. Duan et al., "Electrohydrodynamic directwriting," Nanoscale, vol. 5, no. 24, pp. 12007-12017, 2013.

[22] L. Wang, Y. Luo, Z. Ahmad, J.-S. Li, and M.-W. Chang, "Fabrication of stacked-ring netted tubular constructs via 3D template electrohydrodynamic printing," Journal of Materials Science, vol. 53, no. 17, pp. 11943-11950, 2018.

[23] E. L. Gill, S. Willis, M. Gerigk et al., "Fabrication of designable and suspended microfibers via low-voltage 3D micropatterning," ACS Applied Materials \& Interfaces, vol. 11, no. 22, pp. 1967919690, 2019.

[24] S. Coppola, V. Vespini, G. Nasti et al., "Tethered pyroelectrohydrodynamic spinning for patterning well-ordered structures at micro- and nanoscale," Chemistry of Materials, vol. 26, no. 11, pp. 3357-3360, 2014.

[25] S. Coppola, G. Nasti, M. Todino, F. Olivieri, V. Vespini , and P. Ferraro, "Direct writing of microfluidic footpaths by pyroEHD printing," ACS Applied Materials and Interfaces, vol. 9, no. 19, pp. 16488-16494, 2017.

[26] S. V. Fridrikh, J. H. Yu, M. P. Brenner, and G. C. Rutledge, "Controlling the fiber diameter during electrospinning," Physical Review Letters, vol. 90, no. 14, 2003. 


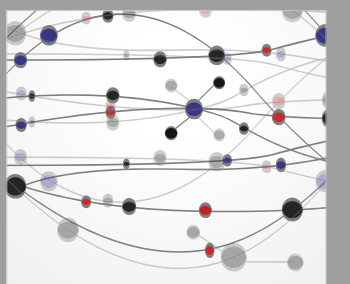

The Scientific World Journal
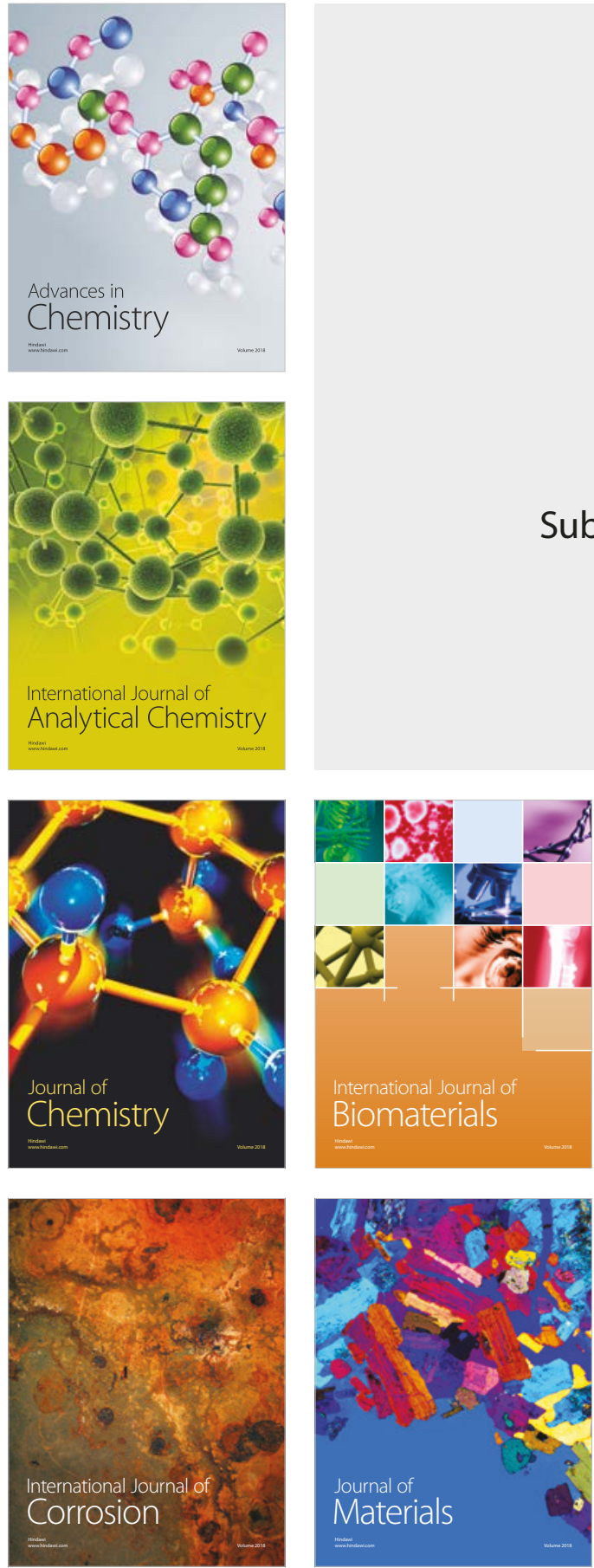

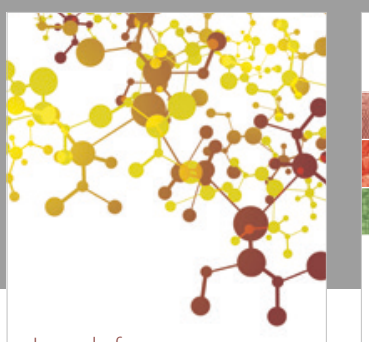

Journal of

Applied Chemistry
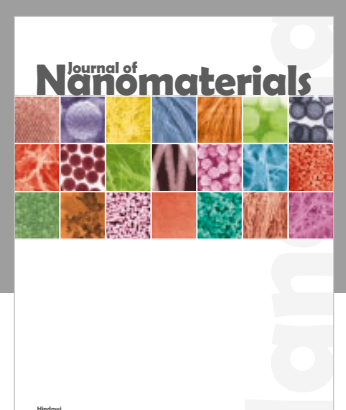

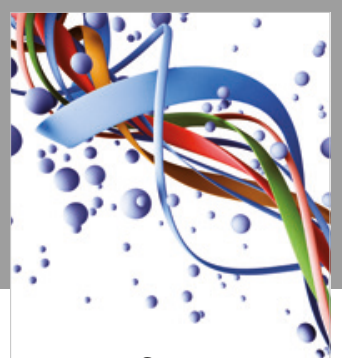

Scientifica

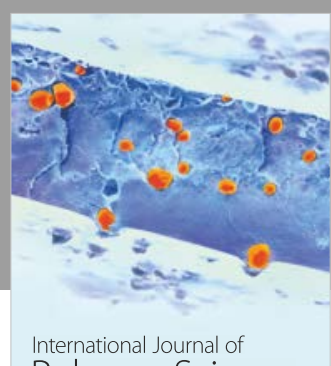

Polymer Science

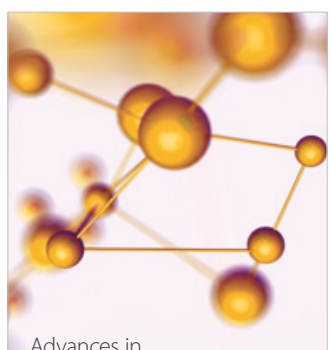

Physical Chemistry
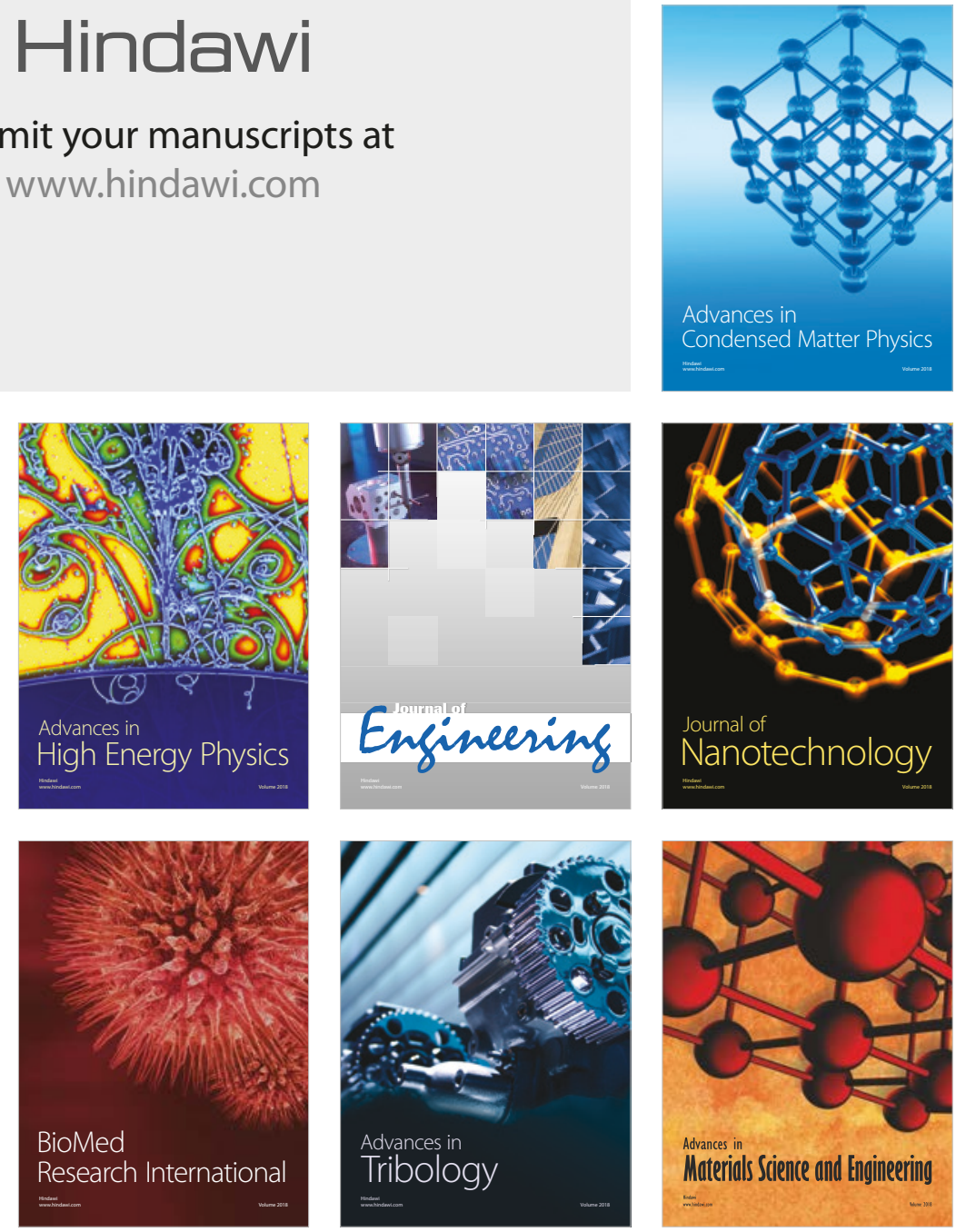